\title{
Cancer Support \& Advice (CANadvice) m- health system for home monitoring and symptom management of patients receiving oral chemotherapy treatment
}

\author{
Miss Drishty Sobnath, Dr Nada Philip, Dr Reem Kayyali, Dr Shereen Nabhani-Gebara \\ Faculty of Science, Engineering and Computing \\ Kingston University \\ London, United Kingdom
}

\begin{abstract}
Introduction: Recent development in digital technology has raised the interest of many researchers in implementing technology in the domain of $m$-health to provide better service to patients. Capecitabine, an oral chemotherapeutic agent causes several side effects which need to be monitored to avoid severe health consequences in patients.

Methodology: A multidisciplinary team of pharmacists, clinicians and developers, was assembled for the design and development of CANadvice system, a mobile android application for patients and a web interface for health care professionals to assist patients in monitoring chemotherapy side effects at home. The application provides real time medical or lifestyle advice to patients and sends alerts to health care professionals for intervention.

Results and Discussion: Two prototypes were designed, implemented and evaluated. Interviews were carried out with 5 health care professionals to investigate the system's effectiveness. The results indicated in this paper recommend using such tool for monitoring side effects. Real time advice and the link to health care professionals were very well accepted.
\end{abstract}

Keywords: m-health, chemotherapy, mobile application, cancer support, canadvice

\section{INTRODUCTION}

M-health is a term used to describe "mobile computing, medical sensor, and communications technologies for health care" [1]. M-health is extensively used today for health services and patient education. There have been many studies evaluating the use of mobile health interventions in health care sector. The benefits of the wireless technology, have been shown in a number of different examples and applications [2] such as to monitor heart rate, blood pressure, blood oximetry and physical activities.

With m-health, patient medical history can be accessed by health-care professionals from anywhere by connecting to the health care hub. Also, laboratory results, pharmaceutical data, insurance information, and other medical resources can be stored on the server for later use. Research carried out by our group concluded that cancer patients need support while at home for medication and to answer drug related queries.

[3][4].

Treatment of cancer includes psychosocial support, surgery, radiotherapy, chemotherapy and hormone therapy that help to extend the patient's life while giving the patient a better quality of life [5]. The use of chemotherapeutic agent, however, can be harmful as they give rise to toxic side effects. In the pharmaceutical world, more and more chemotherapy agents, one of which is capecitabine, are being given to patients in oral form. If side effects of the administered drug are not well monitored, patients' health conditions may be jeopardised, hence immediate treatment is vital.

Capecitabine is an antimetabolite which acts against a number of solid tumors like breast and prostate and is used to relieve pain or distress [5]. In the UK, oral capecitabine is licensed to be used for therapy in several cancer types despite the side effects it can cause, for example in the treatment of gastric cancer, breast cancer, colon or colorectal cancer [6]. It has been observed that lives of patients can be improved by making use of existing technologies in the sense that they can self-monitor their side effects at ease from home, for example, from their smartphone, without the need to visit their health care professional every day. The extent to which mobile technologies is evolving in our history has significantly altered the landscape of many industry sectors. Healthcare sector is one of the sectors that has been influenced by the rapidly rising development of mobile applications and technologies. As described in a review published in BioMedical Engineering OnLine, there is a high potential for mobile or wireless communications to penetrate into society and renovate healthcare and clinical involvement [7]. The number of smartphone users is increasing at a rapid rate and apps are being developed by many health companies to encourage the general public for self-management of certain type of diseases such as asthma, cancer, COPD or cardiovascular diseases. Smartphones are now considered as potential medium in health care management [8]. Hence, the proposed solution was to develop a smart phone application on Android platform to provide real time advice to cancer suffering patients under chemotherapy treatment.

\section{NEED FOR REAL TIME ADVICE}

A systematic review and meta-analysis of the benefits of $\mathrm{m}$ health has shown how mobile health interventions could have beneficial effects on health care delivery processes [9]. Weaver [10] explains how Dikken and Sitzia [11] studied the various 
side effects of the chemotherapy drug and how they badly affect the quality of life of patients. He also discussed the number of toxic death rates caused by colorectal cancer which accounts for 0.8 to $2.2 \%$ (measured by 60 -day all-cause mortality) whereby several side effects like diarrhea, mucositis, febrile neutropenia, vomiting and other toxicities contribute to the death toll above [12]. To minimise the side effects and consequently reduce the death toll, the literature demonstrates that the use of prompt intervention via mobile phones should be used to report early signs of these side effects. Moreover, as stated by Badawi [13], biofeedback sensors, which is used for real time health advisory systems are gradually overtaking traditional methods as they are more beneficial in terms of ubiquity and cost.

A research made by Oxford University has shown a growing demand for the use of mobile phones in the treatment of cancer. Cobern et al [14] described how an experiment was carried out on six colon cancer suffering patients undergoing chemotherapy treatment who had to enter their symptom data on a mobile screen two times a day. The nurses were then alerted by means of a pager and they could deal with the patient. The methods used, however, did not have a history of the side effects experienced by patients. With the CANadvice system, severe side effects experienced by the patients will be automatically recorded in a database which will be accessible by nurses and healthcare professionals.

According to previous studies by our group, it was found out that new approaches were required to help patients self-manage their side effects. The previous study was carried out on 140 adult cancer patients concerning the information they received about their treatment [3-4]. During the analysis, it was found that out of the 140 patients, $7 \%$ of all patients did not recall being advised and $15 \%$ did not remember about having any leaflets about their treatment. Another 12\% were not satisfied with the provided information [4]. It was also mentioned by patients that it was hard sometimes to reach healthcare professionals.

\section{Methodology}

Agile methodology, DSDM (Dynamic systems development methods), based on iterative and incremental development, has been applied during the development of the project. The agile method allows departmentalisations which mean that the work had been divided into smaller groups such as analysis, design, coding, evaluation and testing.

\section{A. System Architecture}

Designers and developers usually suffers from a tension between the development models being approved and the actual design work as they are increasingly expected to deliver high quality systems that fulfil users' needs. The design methodology is guided by three main goals:

- The design must be consistent and logical to patients with no internal contradictions.

- It must cover all the relevant functional requirements.

- The simplest and most smart design must be chosen if more than one design is feasible as elderly patients will be using the application.
There were different phases in the design of this project. The design of the app involved different techniques starting from wireframing, class diagrams, sequence diagrams, design of the database and ending with different prototypes whereby one of them was the final solution to the application. Figure 1 shows the proposed system architecture.

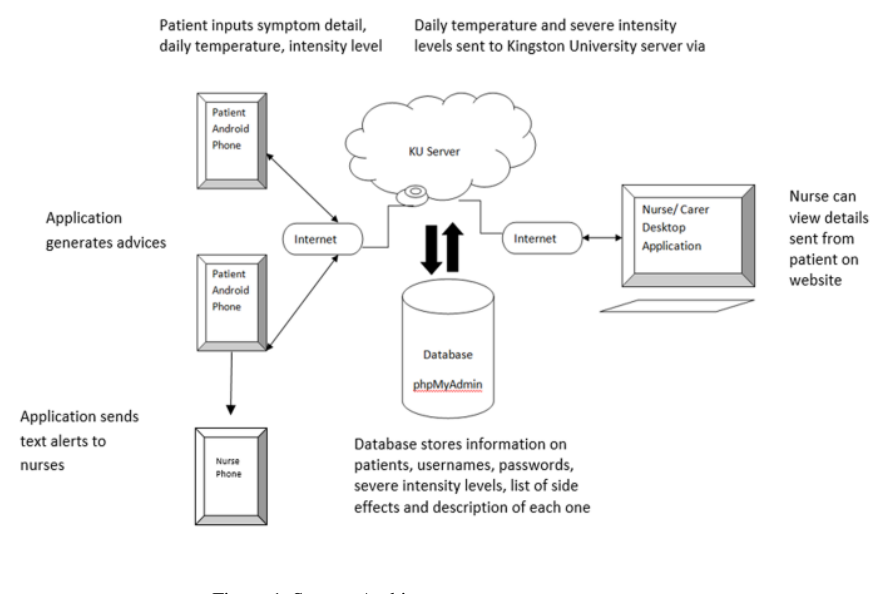

Figure 1 shows the architecture of the CANadvice system demonstrating the different components of the system and how they interact with each other. We have designed the system architecture in a highly modular fashion in order to ease its development and task distribution between the stakeholders.

\section{B. Algorithms}

The mobile application currently displays advice for 9 symptoms and the patient has to choose the intensity level based on their experienced side effects. Patient $\log s$ in and enters daily temperature. He is then asked to select his symptoms from a drop down list. Based on this input, a list of advice is generated for each intensity. Automatic alerts in the form of text messages are sent to registered nurses in case a patient is suffering from severe side effects and needs intervention. The designed screens involving clinical content were based on guidelines [15-16]. Different flowcharts have been used to write the algorithms in the app for each of the 9 side effects.

\section{Prototypes}

During the development of this project, two main prototypes were built based on user requirements. A Visual Prototype (Model) was built to project the anticipated design visual and simulate the appearance, color and images of the proposed product but did not essentially implement the functionalities of the final product. ADT (Android Developer Tools) is a plugin for Eclipse software that provides a collection of tools that are incorporated with the Eclipse IDE (Integrated Development Environment). ADT provides GUI (Graphical User Interface) access to many of the command line SDK (Software Development Kit) tools. It provides a UI (User Interface) design tool for rapid prototyping, designing, and building of mobile application's user-friendly interface. The design tool used was in XML (Extensible Markup Language) format, a format that is 
both human-readable and machine-readable. The first prototype consisted of advice generated for only 4 of the 9 symptoms. The main screens of visual design were shown to the stakeholders. Hence, requested changes were then implemented in the functional prototype model.

A Functional Prototype (Model) was then built. It was a working mobile android application on an actual device used to simulate the final design, content and functionality of the future design. Some early flaws were detected and thus the final product was improved. A major part of an android application's overall lifecycle is an Activity class which represents a single screen with a user interface. The development of the application was done using Eclipse software which allowed the creation of different activities. The whole mobile application was written in Java and XML. The functional prototype generated advice for mild, moderate and severe levels of all 9 main side-effects that could be experienced by patients.

\section{RESUlts}

A full working Android (Version 2.2 Froyo) application was successfully implemented and it provided real time advice to patients. A web application has also been designed and implemented whereby registered nurses and carers can $\log$ in to view patients' details and health history. Figure 2 shows some of the screens of the app. Once the user taps on "Confirm" button, he is prompted to alert his healthcare professional via text messages.

(If $36<$ temp $<37.5$, no error messages will be displayed, else If temp $>37.5$, a warning message will be displayed to say that user has a high temperature and this will be sent to nurses via a text message)
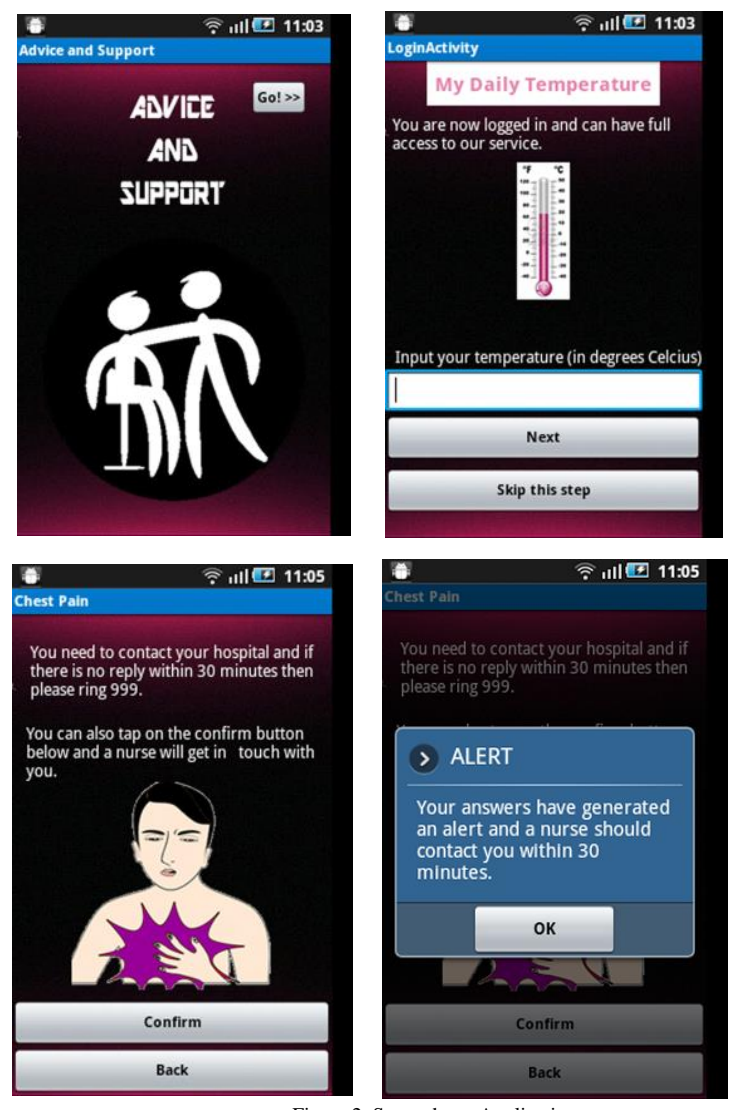

Figure 2. Smartphone Application
In order to log in to the android application and for data to be sent and retrieved from the database, an internet connection needs to be established first. This is done by making HTTP request. The PHP APIs have been written to accept requests by GET/POST methods. After a patient inputs values via the user interface, the data has to be passed from XML format to a language that the server can interpret. This is done by using JSON (JavaScript Object Notation), a lightweight data-interchange format. It is quite easy for developers to read and write. Moreover, machines can easily parse and generate it. JSON has recently become a popular data exchange format and is used in iOS and Android development. It is necessary to change the data format from XML to JSON for efficiency purposes [17]. This is useful for Rich Internet Applications (RIA) as it is a text format that is completely language independent. JSON uses mainly the two structures [17].

- It consists of a collection of name or value pairs. For example, an object, record, dictionary, hash table, keyed list, or associative array.

- It consists of an ordered list of values, for example in the case of an array, vector, list, or sequence.

A file called JSONParser.java was created during the implementation of the project. This parser class uses a method which makes http request to get JSON data and returns a JSONObject. Kingston University student net server has been utilised for this project.

All data sent by the app was securely stored in a database. Via the web interface, health care professionals could have access to patient's symptoms, past history and the date and time that the patient has been suffering from any severe side effect.

\section{A. Evaluation}

The evaluation of the application has been done in two steps. The first prototype was evaluated by S. Ghafoor [18] with 14 cancer patients and survivors recruited through the Macmillan Cancer Voices network. The application was then improved and a second functional prototype was further evaluated with 5 healthcare professionals.

From the first evaluation, it was found that patients would use this application for advice and education as well as for reporting ADRs (Adverse Drug reactions). Patients also wanted to make sure that the alerts transmitted by the application had to be received by somebody competent for intervention. Moreover, information included in the application had to be endorsed by a trustworthy source [18]. The font used and the design was changed to include more pictures and make it more user friendly. The results showed that the use of a smartphone application would be very useful for patients to manage their side effects and to be in touch with healthcare professionals.

The application was then evaluated with 5 healthcare professionals to investigate its effectiveness. The interview was divided into the following sections: (1) Usability Evaluation (To see if the application is user-friendly), (2) Functionality Evaluation (To see if the application is meeting all functional requirements), (3) Patient related questions for evaluation from healthcare professionals.

From the interviews it was found out that, 1) the majority would recommend using such tool for monitoring side effects 
remotely, 2) the application appeared very user-friendly;" Navigating through the app is very good and simple.", 3) severe side effects recorded must be sent to both nurses and the triage system in a hospital, 4) a graph showing the trend in experienced side effect will be beneficial, 5) Patients will feel secure and empowered "The patients will feel more secure as they will know that someone is continuously monitoring their side effects and health care providers will feel better in knowing that there can be a faster response in advising them what to do in case of severe side effects", 6) Mobile technology might not be suitable for elderly patients who are not technologically educated "For patients who have limited knowledge on technological devices, it will be quite hard otherwise it is very easy to navigate through it.". These findings pave the way for the use of the CANadvice application.

\section{B. Testing}

Testing has been done throughout the project lifecycle to investigate, consolidate and refine the whole system so as the best product is delivered to patients. Testing has been done at the end of each iteration especially when an Android activity has been fully implemented. To achieve this, a testing methodology, known as unit testing, has been utilised to check whether the system requirements have been met or not. Using unit testing, test cases have been created to see the output of the mobile application and to get feedback on the usability and suitability of the product. White box method has been used so as any technical problem detected could be debugged by the developer. Dalvik Debug Monitor Service, DDMS, has been used during testing and also during implementation. It is a GUI debugging application which comes with the installed SDK.

\section{CONCLUSION AND FUTURE WORK}

Using mobile phones in the medical field for remote monitoring of symptoms experienced by patients provide an inexpensive solution to traditional ways of using telemedicine. In a conference on Ambient Systems, Networks and Technologies, the set of requirements needed for such a system to be productive was identified: Mobile phone, a web application and a web browser on a phone; accessible by the patient and a user interface for commutations at both sides are the only hardware and software needed for mobile health monitoring. Fewer nurses need to be physically present at the hospitals and the patients do not need to spend much on everyday travelling to have their side effects monitored. This saves cost for hospitalisation and using a mobile phone is a more convenient way to monitor side effects.

The system developed leaves ample room for development of similar disease management system. Missing features could also be added to improve the application as suggested by patients and healthcare professionals during their interviews.

The CANadvice android application integrates a very simple user interface designed for all ages including elderly patients. Both nurses and patients will benefit from the system implemented as no such system currently exists according to research made.
As part of another $\mathrm{PhD}$ study, the application is still being further evaluated and new functionality such as a diary has been added. At the conclusion of this study, a better version of the CANadvice application incorporating new features is being built to make it available on the application market for cancer suffering patients under chemotherapy treatment to improve their everyday life and self-manage their side-effects.

\section{REFERENCES}

1. Istepanian R, Jovanov E, Zhang YT, Introduction to the special section on M-Health: beyond seamless mobility and global wireless health-care connectivity (2004). IEEE Tran INF Technol Biomed 8: 405-414.

2. Istepanian, R.S.H and Wang H, "Telemedicine In UK", in European Telemedicine Glossary of Concepts, Standards, Technologies and Users, Luciano Beolchi (Ed.), 5th Edition, pp. 1159-1165, European Commission Information Society Directorate-General, Brussels (2003)

3. Kayyali R, Nabhani S, Olszweska A, Adeniyi M, Investigation of bowel and breast cancer patients' perception of counselling and written information provided regarding the oral chemotherapy agent capecitabine (2012). IJPP 2: 85.

4. Nabhani-Gebara S, Kayyali R, Olszewska A, Patient Perception of Educational Materiel Surrounding their Cancer treatment. European Journal of Oncology Nursing (2012) ,16: S30

5. WHO, Cancer Control: Diagnosis and Treatment, Key Messages, Geneva: Geneva: World Health Organization (2008), p3.

6. National Institute for Health and Clinical Excellence, Capecitabine for the treatment of advanced gastric cancer (2010), TA191. London: National Institute for Health and Clinical Excellence

7. Boulos, M., Wheeler S., Tavares C, and Jones R, How smartphones are changing the face of mobile and participatory healthcare, BioMedical Engineering Online (2011), (10) p 24. 114.

8. Ackerman, M. J., Filart, R., Burgess, L. P., Lee, I. and Poropatich, R. K., 'Developing Next- Generation Telehealth Tools and Technologies: Patients, Systems, and Data Perspectives', Telemedicine Journal and e-Health (2010), 16 (1), pp. 93-95.

9. Free, C., Phillips, G., Watson, L., Galli, L., Felix, L., Edwards, P.,Haines, A, The effectiveness of mobile-health technologies to improve health care service delivery processes: a systematic review and meta-analysis. PLoS Medicine (2013), 10(1), e1001363. doi:10.1371/journal.pmed.1001363

10. Weaver A,Cobern W, A. M. Young, J. Rowntree, N. Townsend, S. Pearson, J. Smith, O. Gibson, , M. Larsen \& L. Tarassenko (2007), Application of mobile phone technology for managing chemotherapy-associated side-effects, Department of Engineering Science, University of Oxford, UK

11. Dikken C, Sitzia J., Patients' experiences of chemotherapy: sideeffects associated with 5-flourouracil and folinic acid in the treatment of colorectal cancer (1998). Chapter 7 p 371-379.

12. Chau I, Cunningham D, Adjuvant therapy in colon cancer - what, when and how? Annals of Oncology (2006)17: 1347-1359, Published online 8 March 2006

13. Badawi Hawazin, Mohamad Eid and Abdulmotaleb El Saddik, A real-time biofeedback health advisory system for children care, IEEE International Conference on Multimedia and Expo Workshops (2012) , School of Electrical Engineering and Computer Science, University of Ottawa, p 429-434

14. Cobern W, Weaver A, A. M. Young, J. Rowntree, N. Townsend, S. Pearson, J. Smith, O. Gibson, , M. Larsen \& L. Tarassenko, 
Application of mobile phone technology for managing chemotherapy-associated side-effects (2007), Department of Engineering Science, University of Oxford, UK.

15. Cancer.gov, Gastrointestinal Complications - National Cancer Institute (2014). [online] Available at:

http://www.cancer.gov/cancertopics/pdq/supportivecare/gastroin testinalcomplications/HealthProfessional [Accessed: 15 July 2014].

16. Cancer.gov, Nutrition in Cancer Care - National Cancer Institute (2014)[online],Available at:

http://www.cancer.gov/cancertopics/pdq/supportivecare/nutritio n/Patient/page5 [Accessed: 20 July 2014]

17. Ying, M. and Miller, J. 'Refactoring legacy AJAX applications to improve the efficiency of the data exchange component', The Journal of Systems \& Software (2013), 86 (1), pp. 72-88.

18. Ghafoor S, Kayyali R, Nabhani S, Sobnath D and Philip N. (2013), Evaluating patients' acceptability of alternative means of support for oral chemotherapy counselling and side effect management using a smartphone application. Int. J. Pharm. Practice (2013), 21(2): 27-28 